\title{
Parámetros de incubación del starter de Lactobacillus para su aplicación en pan campesino
}

\section{Incubation parameters of the Lactobacillus starter for application in sourdough bread}

\author{
Luis A. Haro ${ }^{1 *}$; Carmen Minaya ${ }^{2}$ \\ 1Lic. Gastronomía y Arte Culinario. Universidad Le Cordon Bleu, Facultad de Ciencias de los Alimentos. Lima, Perú; e-mail: 2018i00148@ulcb.edu.pe; \\ (D) https://orcid.org/0000-0001-7355-6243 \\ ${ }^{2}$ Ing. Alimentario, Mg.Sc. Universidad Le Cordon Bleu, Facultad de Ciencias de los Alimentos. Lima, Perú; e-mail: carmen.minaya@ulcb.edu.pe; \\ (D) https://orcid.org/0000-0002-4087-9422 \\ *autor para correspondencia: luishf2005@hotmail.com
}

Cómo citar: Haro, L.A.; Minaya, C. 2020. Parámetros de incubación del starter de Lactobacillus para su aplicación en pan campesino. Rev. U.D.C.A Act. \& Div. Cient. 23(2):e1742. http://doi.org/10.31910/rudca.v23.n2.2020.1742

Artículo de acceso abierto publicado por Revista U.D.C.A Actualidad \& Divulgación Científica, bajo una licencia Creative Commons CC BY-NC 4.0

Publicación oficial de la Universidad de Ciencias Aplicadas y Ambientales U.D.C.A, Institución de Educación Superior Acreditada de Alta Calidad por el Ministerio de Educación Nacional.

Recibido: Octubre 19 de 2020 Aceptado: Diciembre 1 de 2020 Editado por: Rita María Ávila G. de Hernández

\section{RESUMEN}

El fermento natural o starter es un insumo panadero, que se ha revalorizado, en la actualidad, por sus beneficios y por su aporte de sabor. En este estudio, se determinaron los parámetros de cultivo del starter espontáneo de Lactobacillus, a partir de harina integral, para aplicarlo en la elaboración de pan campesino, en función de sus características sensoriales y mecánicas. Se elaboraron tres starters, para lo cual, se mezcló harina integral con agua, en una proporción de 45,5 y $54,5 \%$, respectivamente; posterior a ello, se fermentaron, espontáneamente, a 20,24 y $28^{\circ} \mathrm{C}$, hasta un $\mathrm{pH}$ final de 4 , a partir de los cuales, se elaboraron panes campesinos. Los panes fueron evaluados sensorialmente, por un panel de expertos (07), mediante una escala lineal no estructurada de 5 puntos, en cuanto a la forma ovalada, apariencia de la costra, masticabilidad de miga, gusto ácido y apariencia de los alveolos. El pan elaborado con el fermento a $24^{\circ} \mathrm{C}$ fue seleccionado por los panelistas, debido a que presentó puntajes superiores $(\mathrm{p}<0,05)$, en los atributos de apariencia de la costra $(4,24)$, masticabilidad de la miga $(5,0)$, gusto ácido $(4,67)$ y apariencia de los alveolos (4,0). El fermento empleado presentó 79 x $10^{7} \mathrm{UFC} \mathrm{g}^{-1}$ de bacterias acidolácticas. Finalmente, la textura del pan campesino seleccionado fue caracterizada instrumentalmente en términos de dureza $(102,09 \mathrm{~N})$, gomosidad $(43,02 \mathrm{~N})$, masticabilidad $(37,36 \mathrm{~N})$, elasticidad $(0,87)$ y cohesividad $(0,42)$, perfil que se puede emplear como parámetro de calidad, para pan campesino y otras variedades similares.

Palabras clave: Análisis sensorial; Fermento natural; Pan campesino; Perfil de textura instrumental.

\section{ABSTRACT}

The natural ferment or sourdough starter is a bakery input that has been revalued nowadays for its benefits and flavour improve. 
In this study, the incubation parameters of the spontaneous Lactobacillus starter made with wholemeal flour were determined to be applied in sourdough bread production depending on its sensory and mechanical characteristics. Three starters were made with wholemeal flour mixed with water in a proportion of 45.5 and $54.5 \%$ respectively, then were fermented spontaneously at 20, 24 and $28^{\circ} \mathrm{C}$ until a final $\mathrm{pH}$ of 4 , from which sourdough breads were produced. The breads were sensory evaluated by a panel of experts (07), using a 5-point unstructured linear scale, in terms of oval shape, crust appearance, crumb chewiness, sourness and alveoli appearance. The bread made with the starter at $24^{\circ} \mathrm{C}$ was selected because of the higher scores $(\mathrm{p}<0.05)$ in the attributes of crust appearance (4.24), crumb chewiness (5.0), sourness (4.67) and alveoli appearance (4.0). The starter used exhibit $79 \times 10^{7} \mathrm{CFU} \mathrm{g}{ }^{-1}$ of lactic acid bacteria. Finally, the texture of the selected sourdough bread was characterized in terms of hardness $(102.09 \mathrm{~N})$, gumminess $(43.02 \mathrm{~N})$, chewiness $(37.36 \mathrm{~N})$, springiness $(0.87)$ and cohesiveness $(0.42)$, a profile that can be used as a quality parameter for sourdough bread and other similar varieties.

Keywords: Sensory evaluation; Sourdough bread; Sourdough; Texture profile analysis.

\section{INTRODUCCIÓN}

Los patrones de consumo, en la actualidad, muestran una inclinación por comprar y promover productos menos industrializados en todas las categorías de alimentos, como carnes, frutas, verduras y panes (Si, 2020); esta última, teniendo, como agente principal, el empleo de starters o fermentos naturales.

Estos fermentos, se utilizan, usualmente en la panadería artesanal, como símbolo de calidad y, recientemente, están captando la atención del mercado, en países donde las tradiciones panaderas fueron sustituidas por la automatización y poca regulación acerca del uso de starters (Gänzle, 2014).

El fermento natural (conocido comúnmente como masa madre o starter) es definido como "una mezcla de harina de trigo y/o centeno, agua, eventualmente con sal agregada y fermentada de manera espontánea" (Corsetti, 2013). En esta masa, se encuentra un complejo ecosistema de levaduras y bacterias endógenas, que conviven en simbiosis, siendo Lactobacillus sanfranciscensis, el microorganismo identificado en la mayoría de los fermentos y, en menor medida, levaduras salvajes (De Angelis et al. 2001; Hammes \& Gänzle, 1998). Ambos microorganismos, se hallan de forma natural en el grano de trigo, confiriendo al pan, un apreciado aroma y sabor ácido, producto de la fermentación (Chavan \& Chavan, 2011).

La complejidad sensorial del pan de masa madre o artesanal, se atribuye a los diferentes compuestos volátiles (Gänzle \& Gobbetti, 2013; Gobbetti et al. 1995), que no se encuentran en panes comerciales (Decock \& Cappelle, 2005). Sumado a ello, nutricionalmente presenta mejor digestibilidad (Rizzello et al. 2019), actividad fitasa (De Angelis et al. 2003), potencial de tolerancia al gluten por parte de celiacos (Di Cagno et al. 2004) y liberación de compuestos fenólicos (Montemurro et al. 2020). Todos estos factores, en sinergia con la acidificación de la masa, provocan un alargamiento de la vida útil y resistencia a microorganismos patógenos, debido al descenso del pH (Gerez et al. 2009), además de su capacidad antimicótica y reducción de micotoxinas, produciendo un alimento más seguro (Hassan et al. 2016).

Todos los beneficios nutricionales, tecnológicos y organolépticos son resultado de los parámetros de incubación de la masa madre, como la temperatura que, según Katina (2005), debe fluctuar entre 22 a $24^{\circ} \mathrm{C}$; el pH entre 3,5 - 4 (Clarke et al. 2004; Collar et al. 1994) y un recuento de bacterias acidolácticas de $10^{7}$ a $10^{9}$ de UFC g ${ }^{-1}$ (Montville \& Matthews, 2009). Estos factores influyen positivamente en las evaluaciones sensoriales del aspecto, tanto exterior como interior, si van de la mano con una larga fermentación (Brabant et al. 2007) y se pueden medir objetivamente, a través de un análisis perfil de textura instrumental (Swelim et al. 2014).

A pesar de que en la literatura se encuentran los criterios para obtener un starter panadero de Lactobacillus, existe mucha variabilidad, como efecto de las temperaturas de cultivo, así como el sustrato, $\mathrm{pH}$, potencial redox, factores endógenos, variedad del trigo, actividad de agua, entre otros (De Vuyst \& Vancanneyt, 2007; Zhang et al. 2019), sumado a que muchos autores elaboran el fermento a partir de una cepa específica, pero no de la fermentación espontánea de la harina, encontrándose, incluso, starters elaborados con otras harinas, como la de quinua (Rizzello et al. 2016). Producto de esto y de la poca estandarización de las variables surgen múltiples problemas, al querer generar fermentos, como una baja calidad fermentativa, acidez desagradable o una nula presencia de microorganismos acidolácticos.

Debido a lo expuesto, el objetivo de esta investigación fue determinar los parámetros de cultivo del starter espontáneo de Lactobacillus, a partir de harina integral y aplicarlo en la elaboración de pan campesino, en función de sus características sensoriales y mecánicas. Esto servirá como punto de inicio para otras variantes similares de este pan, además de aprovechar sus beneficios sensoriales, nutricionales y tecnológicos.

\section{MATERIALES Y MÉTODOS}

Materiales. La harina integral ("Ayni”), empleada en la elaboración de los starters y en el pan campesino, fue orgánica y producida en el valle del Mantaro, Junín, a 3.300m s.n.m. Asimismo, se empleó harina panadera, con un $12 \%$ de proteínas, indicado en la información nutricional.

Elaboración de los starters. Se empleó harina integral de trigo, por la alta actividad fermentativa (Sánchez, 2003) y se mezcló con agua, en una proporción de 45,5 y 54,5\%, respectivamente, lo que, en porcentaje panadero, indica una hidratación del 119,78\%. Se dio una textura homogénea para un desarrollo más uniforme y difusión de los ácidos orgánicos (Clarke et al. 2003). Posteriormente, las mezclas se incubaron a 20,24 y $28^{\circ} \mathrm{C}$, en una cámara de fermentación (METRO Flavour View C175 Heated Cabinet) y se monitoreó por triplicado la acidez, a cada hora, por medio de un potenciómetro 
(marca "Kyntel ATC-2011). Del mismo modo, se contó el tiempo en que cada muestra llegase a un $\mathrm{pH}$ final de 4 y que presentasen burbujas propias de la fermentación, debido a que es un indicador de actividad microbiológica (Chavan \& Chavan, 2011; Lhomme et al. 2015).

Elaboración del pan campesino. Se elaboró panes campesinos con los tres fermentos obtenidos, codificados como P20, P24 y P28; para ello, como primera etapa, se empleó una formulación estándar similar al pan comercial, compuesta por harina panadera $(750 \mathrm{~g})$, harina integral $(250 \mathrm{~g})$, agua $(680 \mathrm{~mL})$, starter $(250 \mathrm{~g})$ y sal $(25 \mathrm{~g})$. Se empleó integral, para incrementar la biodisponibilidad de minerales
(Karaman et al. 2018) y se utilizó un 25\% de fermento, con respecto al peso de la harina total (Brandt, 2007).

Para el procesamiento, se siguió las etapas mostradas en la figura 1. Se empleó una máquina amasadora (marca Nova KN50) y una cámara de fermentación (marca METRO Flavor View C175 Heated Cabinet), para el fermentado final $\left(28^{\circ} \mathrm{C}\right)$; por último, el horneado (en dos tiempos), se hizo en un horno de convección forzada (marca Rational SCC 101G). Cabe señalar que durante el proceso se tuvo una temperatura ambiental de $26^{\circ} \mathrm{C}$, humedad relativa de $78 \%$ y la forma adoptada fue la de pan bâtard francés.

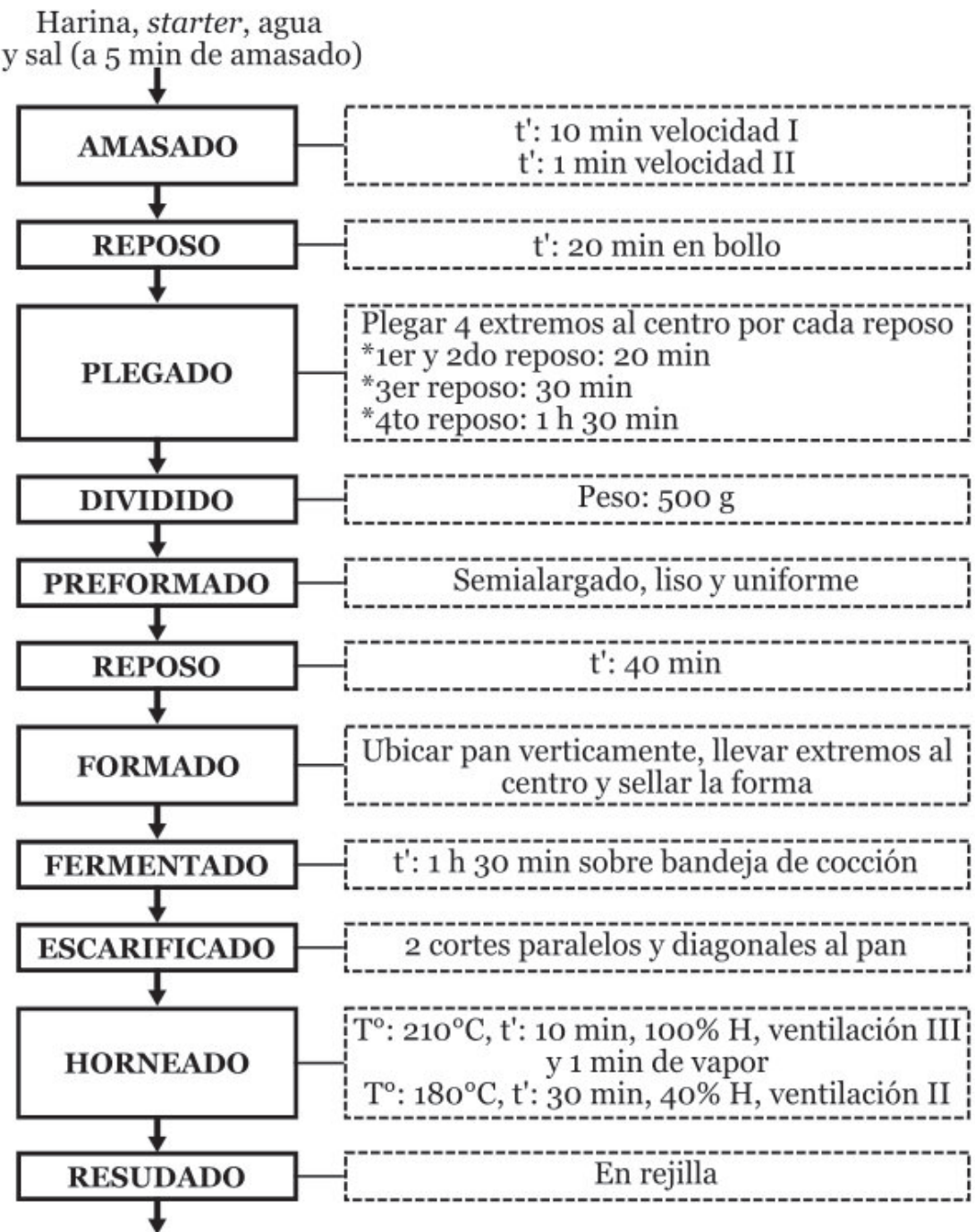

Pan campesino

Figura 1. Flujo de elaboración de pan campesino.

\section{Evaluación sensorial}

Selección y conceptualización de atributos sensoriales. Se elaboró una ficha descriptiva, mediante el método QDA, como detalla Stone \& Sidel (2004), empleando un panel experto compuesto por siete especialistas en panadería; se tomaron en cuenta los factores externos e internos (Brabant et al. 2007), que caracterizan al pan campesino, tomándose como referencia uno comercial (Tabla 1). Esta ficha sirvió como complemento a la evaluación sensorial. 
Tabla 1. Definiciones de los atributos sensoriales empleados para el análisis.

\begin{tabular}{|c|c|c|c|}
\hline \multicolumn{2}{|c|}{ Atributo sensorial } & Valoración & Descripción \\
\hline \multirow{4}{*}{ Externo } & \multirow[t]{2}{*}{ Forma ovalada } & Malo & Deforme e irregular; no presenta extremos ovalados. \\
\hline & & Excelente & Ovalado en todos 4 extremos. \\
\hline & \multirow{2}{*}{$\begin{array}{l}\text { Apariencia de la } \\
\text { costra }\end{array}$} & Malo & $\begin{array}{c}\text { Marrón con tendencia a negro en todo el pan, muy dura y muy } \\
\text { gruesa. }\end{array}$ \\
\hline & & Excelente & $\begin{array}{c}\text { Marrón dorado uniforme en toda superficie del pan, crocante y } \\
\text { gruesa. }\end{array}$ \\
\hline \multirow{6}{*}{ Interno } & \multirow{2}{*}{$\begin{array}{l}\text { Masticabilidad } \\
\text { de miga }\end{array}$} & Malo & $\begin{array}{l}\text { Alta resistencia al masticar para la desintegración y posterior } \\
\text { deglución ( } 41-44 \text { masticadas). }\end{array}$ \\
\hline & & Excelente & $\begin{array}{l}\text { Fácil masticación para la desintegración y posterior deglución } \\
\qquad(<28 \text { masticadas }) \text {. }\end{array}$ \\
\hline & \multirow[b]{2}{*}{ Gusto ácido } & Malo & Acidez de fermentación intensa; intolerable y desagradable. \\
\hline & & Excelente & $\begin{array}{l}\text { Ligera acidez de fermentación de fondo, acompañado de } \\
\text { sabores y aromas agradables. }\end{array}$ \\
\hline & \multirow{2}{*}{$\begin{array}{l}\text { Apariencia de la } \\
\text { miga: alveolos }\end{array}$} & Malo & $\begin{array}{l}\text { Alveolos deformes, irregulares y asimétricos; muy pequeños y } \\
\text { tupidos. Miga muy densa. }\end{array}$ \\
\hline & & Excelente & $\begin{array}{l}\text { Alveolos de tamaño mediano }(1,1-2 \mathrm{~cm} \text { máximo }) \text { y } \\
\text { uniformidad en el tamaño. Miga muy ligera. }\end{array}$ \\
\hline
\end{tabular}

Análisis sensorial. Se realizó la evaluación sensorial de los panes elaborados, mediante un QDA, según las especificaciones dadas por INDECOPI (2008), con un jurado experto, conformado por siete personas, quienes evaluaron por duplicado los cinco atributos esenciales, descritos anteriormente. Para ello, marcaron un aspa sobre una línea de $10 \mathrm{~cm}$; luego, se realizó una transformación, para establecer una escala de 5 puntos, como refiere Swelim et al. (2014). Por medio de este análisis, se determinó el pan con mayor calificación y, por ende, la temperatura de cultivo de un fermento natural para panadería, para los posteriores análisis.

\section{Caracterización del pan y fermento seleccionado}

Evaluación microbiológica del fermento. El recuento de Lactobacillus al fermento seleccionado fue determinado de acuerdo con la American Public Health Association (2015) y el resultado se expresó en unidades formadoras de colonias por gramo de starter.

Evaluación por texturómetro del pan campesino. El perfil de textura del pan de mayor puntaje fue realizado por un texturómetro "Instron 3365, celda carga: 5KN", con un accesorio Compression anvil, 4IN. Square área (S5402A), 57mm de diámetro. Se evaluó la dureza, la cohesividad, la elasticidad, la gomosidad y la masticabilidad de 6 tajadas de $20 \mathrm{~mm}$ de espesor, a una velocidad de ensayo de $0.50 \mathrm{~mm}$ $\mathrm{s}^{-1} \mathrm{y}$ a una temperatura promedio de $24^{\circ} \mathrm{C}$.
Análisis estadístico. Para analizar los puntajes obtenidos en la evaluación sensorial del QDA, se utilizó el DCA por cada pan, el ANOVA para ver el grado de significancia de las muestras además de la prueba de Tukey, para evaluar la diferencia entre las medias. Tanto ANOVA como Tukey, a un nivel de confianza, del 95\%.

\section{RESULTADOS Y DISCUSIÓN}

Parámetros de incubación del starters. La incubación de los starters a 20,24 y $28^{\circ} \mathrm{C}$ tuvieron una duración de 71,54 y 36 horas, respectivamente. Estos presentaron un $\mathrm{pH}$ inicial de 5,85, con pico de 6,1, luego de $10 \mathrm{~h}$, aproximadamente, pero, posterior a ello comenzó, el descenso hasta un pH final de 4 (Figura 2).

La velocidad promedio en el cultivo a $28^{\circ} \mathrm{C}$ fue mayor que todas, con 0.0531 de descenso de $\mathrm{pH}$ por hora (Tabla 2).

Quellen-Field (2012) menciona que un starter crece entre 20 y $28^{\circ} \mathrm{C}$ e, incluso, hasta $30^{\circ} \mathrm{C}$ (Clarke et al. 2003), lo que fue corroborado durante la investigación, al usar temperaturas de incubación de 20,24 y $28^{\circ} \mathrm{C}$, observándose en las tres, capacidad fermentativa; sin embargo, el aporte de sabor y aromas es mayor mientras la temperatura es más baja (alrededor de $23 \pm 1^{\circ} \mathrm{C}$ ), por el aporte de compuestos aromáticos (Katina, 2005). Al realizarse las evaluaciones sensoriales, los panes elaborados, empleando un starter a $24^{\circ} \mathrm{C}$, 


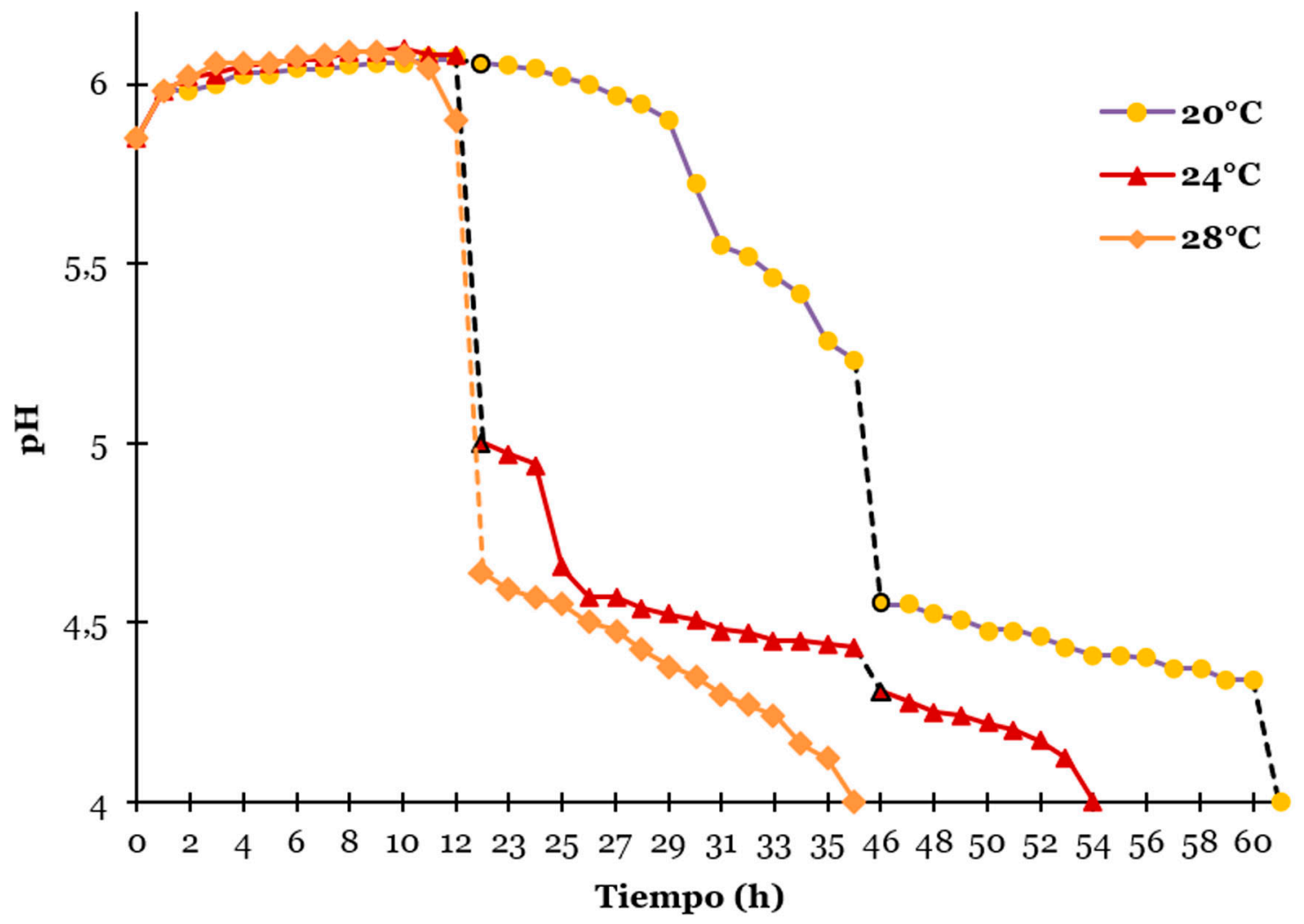

Figura 2. Perfil del pH en los tres fermentos durante la incubación.

*Las líneas punteadas indican intervalos de 10 horas, en las cuales, no se pudo tomar las mediciones, por acceso a las cámaras.

Tabla 2. Efectos de distintas temperaturas de incubación.

\begin{tabular}{|c|c|c|c|c|}
\hline \multirow{2}{*}{ Temperatura $\left({ }^{\circ} \mathbf{C}\right)$} & \multicolumn{2}{|c|}{$\mathbf{p H}$} & \multirow{2}{*}{ Tiempo (h) } & \multirow{2}{*}{$\Delta \mathbf{p H ~ h}^{-1} *$} \\
\cline { 4 - 5 } & Inicial & Final & & \\
\hline 20 & \multirow{3}{*}{5,85} & \multirow{2}{*}{4,00} & 71 & $0,0369 \pm 0,05$ \\
\hline 24 & & & 54 & $0,0392 \pm 0,06$ \\
\hline & & 36 & $0,0531 \pm 0,04$ \\
\hline
\end{tabular}

*Promedio calculado desde el momento que llegó al pico de pH y comenzó su descenso.

evidenciaron que es la temperatura sensorialmente aceptable para el cultivo, dado que obtuvo el mayor puntaje, en cuatro de los cinco atributos, mediante el ANOVA y la prueba Tukey.

Se comprobó que los fermentos, al llegar a un $\mathrm{pH}$ de 4, presentaron actividad fermentativa, reflejándose en un mayor volumen por la producción de $\mathrm{CO}_{2}$; es posible, también, extender el tiempo de fermentación en los starters hasta lograr un $\mathrm{pH}$ menor. Cabe mencionar que un $\mathrm{pH}$ menor a 4 es importante para inhibir y reducir bacterias patógenas provenientes por contaminación, como Escherichia coli, Enterococcus sp., entre otros (Boreczek et al. 2020).

Evaluación sensorial. Como resultado de la evaluación sensorial, se observó diferencias significativas $(p<0,05)$, en cuatro de los atributos evaluados (Tabla 3), siendo los tratamientos P20 y P24 similares en solo un atributo (forma ovalada), P24 y P28 en tres atributos (apariencia de la costra, masticabilidad de la miga y gusto ácido) y todas similares, en cuanto a la apariencia de la miga.

Debido a que el tratamiento P24 comparado con P28 presentó mayor puntaje en cuatro de los cinco atributos, fue escogido como el mejor y se procedió a caracterizar, tanto el fermento empleado como el pan elaborado con éste.

Según lo reportado para la evaluación de panes con starter, el color de la costra, la forma, la porosidad, la acidez, el retrogusto, la homogeneidad en la miga, entre otros, son tomados en cuenta (Brabant et al. 2007; Venturi et al. 2016). Por ello, se consideró, del 
Tabla 3. Puntajes en los atributos sensoriales de los panes campesino.

\begin{tabular}{|l|c|c|c|c|}
\hline \multirow{2}{*}{ Atributo sensorial } & \multirow{2}{*}{ Pan comercial* } & \multicolumn{3}{|c|}{ Panes campesinos } \\
\cline { 3 - 5 } & & P20 & P24 & P28 \\
\hline Forma ovalada & 4,0 & $2,84 \pm 0,62^{\mathrm{a}}$ & $3,41 \pm 0,60^{\mathrm{a}}$ & $3,71 \pm 0,49^{\mathrm{b}}$ \\
\hline Apariencia de la costra & 4,7 & $3,14 \pm 0,52^{\mathrm{a}}$ & $4,24 \pm 0,38^{\mathrm{b}}$ & $4,07 \pm 0,45^{\mathrm{b}}$ \\
\hline Masticabilidad de miga & 4,5 & $4,79 \pm 0,21^{\mathrm{a}}$ & $5,00 \pm 0,00^{\mathrm{b}}$ & $5,00 \pm 0,00^{\mathrm{b}}$ \\
\hline Gusto ácido & 5,0 & $3,74 \pm 0,63^{\mathrm{a}}$ & $4,67 \pm 0,37^{\mathrm{b}}$ & $4,43 \pm 0,53^{\mathrm{b}}$ \\
\hline $\begin{array}{l}\text { Apariencia de la miga: } \\
\text { alveolos }\end{array}$ & 4,1 & $3,49 \pm 0,71^{\mathrm{a}}$ & $4,00 \pm 0,25^{\mathrm{a}}$ & $3,43 \pm 0,53^{\mathrm{a}}$ \\
\hline
\end{tabular}

"No fue evaluado por los 7 jurados; ${ }^{\text {a b }}$ Medias en las filas para cada atributo, con diferentes superíndices, indica una diferencia significativa $(\mathrm{p}<0,05)$.

mismo modo, atributos similares, para obtener las cualidades más importantes, en un pan campesino.

Asimismo, los componentes volátiles y, por ende, las cualidades sensoriales, van estrechamente ligadas a la temperatura de fermentación en panadería, como lo referido por Birch et al. (2013); por esa razón, una temperatura intermedia de fermentación ayudaría a abarcar los atributos considerados deseables, sin dejar de lado los tecnológicos, como el tiempo de proceso. Se pudo comprobar que un starter cultivado a $24^{\circ} \mathrm{C}$ cumple con la mayoría de los atributos sensoriales, sin hacer muy extenso el tiempo de la obtención de éste. Del mismo modo, se podría emplear mezclas de distintos starters, para mejorar el perfil organoléptico (Gül et al. 2005).

Evaluación microbiológica del starters. El fermento de mayor puntaje sensorial (cultivado a $24^{\circ} \mathrm{C}$ ) presentó un recuento total de Lactobacillus de $79 \times 10^{7} \mathrm{UFC} \mathrm{g}^{-1}$, encontrándose en el rango de $10^{7}$ a $10^{9}$ de $\mathrm{UFC} \mathrm{g}^{-1}$ de BAL (bacterias ácido lácticas), descrito por Montville \& Matthews (2009). Es muy probable que la bacteria dominante de este cultivo sea Lactobacillus sanfranciscensis, en simbiosis con la levadura Candida milleri (Lhomme et al. 2015; Venturi et al. 2012; Zhang et al. 2019).

Perfil de textura instrumental del pan. Los promedios de los valores del perfil de textura a un pan campesino con $25 \%$ de harina integral, elaborado con starter, a $24^{\circ} \mathrm{C}$, fueron $102,09 \mathrm{~N}$ de dureza, $43,02 \mathrm{~N}$ de gomosidad, $37,36 \mathrm{~N}$ de masticabilidad, 0,87 de elasticidad y 0,42 de cohesividad.

Se observa que los mayores valores se muestran en la dureza, que va ligado a la costra del pan; gomosidad, que va acorde con la miga del pan, que es considerada semigomosa y masticabilidad, que relaciona la dureza, la elasticidad y la cohesividad. Omedi \& Huang (2016) reportaron un valor menor elasticidad, similar en cuanto a la masticabilidad, pero superiores en dureza, gomosidad y cohesividad, para panes campesinos con Lactobacillus plantarum y Lactobacillus sanfranciscensis, lo cual, se puede deber a que la tasa de hidratación durante la investigación fue elevada.
Del presente estudio, se puede resaltar, que la elaboración de panes campesinos semi integrales empleando un starter con harina integral, preparado a $24^{\circ} \mathrm{C}$, ofrece ventajas sensoriales frente a aquellos incubados a 20 y $28^{\circ} \mathrm{C}$, teniendo una dureza, gomosidad, masticabilidad, elasticidad y cohesividad de $102.09 \mathrm{~N}, 43.02 \mathrm{~N}$, $37.36 \mathrm{~N}, 0.87$ y 0.42 , respectivamente. Además, el fermento a estas condiciones presenta $79 \times 10^{7}$ de $\mathrm{UFC} \mathrm{g}^{-1}$, que se podría utilizar para elaborar distintas variedades de pan, para mejorar su perfil nutricional, sensorial y vida útil.

Agradecimientos. Los autores agradecen a los panelistas, por su colaboración en la evaluación sensorial y, en especial, a Olivier Rousseau, de Le Cordon Bleu, por su guía técnica constante, durante la producción de los panes. Conflictos de interés: El manuscrito fue preparado y revisado con la participación de los autores, quienes declaran que no existe ningún conflicto de intereses que ponga en riesgo la validez de los resultados presentados.

\section{REFERENCIAS}

1. AMERICAN PUBLIC HEALTH ASSOCIATION. 2015. Salad Dressings. En: Salfinger, Y.; Lou Tortorello, M. (Eds.), Compendium of Methods for the Microbiological Examination of Foods (1st ed.). American Public Health Association. (Estados Unidos). https://doi.org/10.2105/MBEF.0222

2. BIRCH, A.N.; PETERSEN, M.A.; HANSEN, Å.S. 2013. The aroma profile of wheat bread crumb influenced by yeast concentration and fermentation temperature. LWT - Food Science and Technology. (Estados Unidos). 50(2):480-488. https://doi.org/10.1016/j.lwt.2012.08.019

3. BORECZEK, J.; LITWINEK, D.; ŻYLIŃSKA-URBAN, J.; IZAK, D.; BUKSA, K.; GAWOR, J.; GROMADKA, R.; BARDOWSKI, J.K.; KOWALCZYK, M. 2020. Bacterial community dynamics in spontaneous sourdoughs made from wheat, spelt, and rye wholemeal flour. 
MicrobiologyOpen. (Estados Unidos). 9(4):1-13.

https://doi.org/10.1002/mbo3.1009

4. BRABANT, C.; FOSSATI, D.; KLEIJER, G.; VINCENT, V.; JACQUES, C. 2007. Influence de la variété de blé sur le goût du pain. Revue Suisse d'Agriculture. (Suiza). 39(3):101-108.

5. BRANDT, M.J. 2007. Sourdough products for convenient use in baking. Food Microbiology. (Estados Unidos). 24(2):161164

https://doi.org/10.1016/j.fm.2006.07.010

6. CHAVAN, R.S.; CHAVAN, S.R. 2011. Sourdough Technology-A Traditional Way for Wholesome Foods: A Review. Comprehensive Reviews in Food Science and Food Safety. (Estados Unidos). 10(3):169-182. https://doi.org/10.1111/j.1541-4337.2011.00148.x

7. CLARKE, C.; SCHOBER, T.J.; ANGST, E.; ARENDT, E.K. 2003. Use of response surface methodology to investigate the effects of processing conditions on sourdough wheat bread quality. European Food Research and Technology. (Alemania). 217(1):23-33. https://doi.org/10.1007/s00217-003-0724-1

8. CLARKE, C.; SCHOBER, T.J.; DOCKERY, P.; O'SULLIVAN, K.; ARENDT, E.K. 2004. Wheat Sourdough Fermentation: Effects of Time and Acidification on Fundamental Rheological Properties. Cereal Chemistry. (Estados Unidos). 81(3):409-417. https://doi.org/10.1094/CCHEM.2004.81.3.409

9. COLLAR, C.; BENEDITO DE BARBER, C.; MARTÍNEZANAYA, M.A. 1994. Microbial Sour Doughs Influence Acidification Properties and Breadmaking Potential of Wheat Dough. J. Food Science. (Estados Unidos). 59(3):629-633.

https://doi.org/10.1111/j.1365-2621.1994.tb05579.x

10. CORSETTI, A. 2013. Technology of sourdough fermentation and sourdough applications. En: Handbook on Sourdough Biotechnology. Springer US. (Estados Unidos). p.85-103. https://doi.org/10.1007/978-1-4614-5425-0_4

11. DE ANGELIS, M.; BINI, L.; PALLINI, V.; COCCONCELLI, P.S.; GOBBETTI, M. 2001. The acid-stress response in Lactobacillus sanfranciscensis CB1. Microbiology. (Reino Unido). 147(7):1863-1873.

https://doi.org/10.1099/00221287-147-7-1863

12. DE ANGELIS, M.; GALLO, G.; CORBO, M.R.; MCSWEENEY, P.L.H.; FACCIA, M.; GIOVINE, M.; GOBBETTT, M. 2003. Phytase activity in sourdough lactic acid bacteria: Purification and characterization of a phytase from
Lactobacillus sanfranciscensis CB1. International J. Food Microbiology. (Países Bajos). 87(3):259-270.

https://doi.org/10.1016/S0168-1605(03)00072-2

13. DE VUYST, L.; VANCANNEYT, M. 2007. Biodiversity and identification of sourdough lactic acid bacteria. Food Microbiology. (Estados Unidos). 24(2):120-127. https://doi.org/10.1016/j.fm.2006.07.005

14. DECOCK, P.; CAPPELLE, S. 2005. Bread technology and sourdough technology. En: Handbook on Sourdough Biotechnology (Vol. 16, Issues 1-3). Springer US. (Estados Unidos). p.113-120. https://doi.org/10.1016/j.tifs.2004.04.012

15. DI CAGNO, R.; DE ANGELIS, M.; AURICCHIO, S.; GRECO, L.; CLARKE, C.; DE VINCENZI, M.; GIOVANNINI, C.; D'ARCHIVIO, M.; LANDOLFO, F.; PARRILLI, G.; MINERVINI, F.; ARENDT, E.; GOBBETTI, M. 2004. Sourdough Bread Made from Wheat and Nontoxic Flours and Started with Selected Lactobacilli Is Tolerated in Celiac Sprue Patients. Applied and Environmental Microbiology. (Estados Unidos). 70(2):1088-1096. https://doi.org/10.1128/AEM.70.2.1088-1096.2004

16. GÄNZLE, M. 2014. Bread: Sourdough Bread. En: Encyclopedia of Food Microbiology: Second Edition (Vol. 1). Academic Press. (Estados Unidos). p.309-315. https://doi.org/10.1016/B978-0-12-384730-0.00045-8

17. GÄNZLE, M.; GOBBETTI, M. 2013. Physiology and biochemistry of lactic acid bacteria. En: Handbook on Sourdough Biotechnology. Springer US. (Estados Unidos). p.183-216. https://doi.org/10.1007/978-1-4614-5425-0_7

18. GEREZ, C.L.; TORINO, M.I.; ROLLÁN, G.; FONT DE VALDEZ, G. 2009. Prevention of bread mould spoilage by using lactic acid bacteria with antifungal properties. Food Control. (Paises Bajos). 20(2):144-148. https://doi.org/10.1016/j.foodcont.2008.03.005

19. GOBBETTI, M.; SIMONETTI, M.; CORSETTI, A.; SANTINELLI, F.; ROSSI, J.; DAMIANI, P. 1995. Volatile compound and organic acid productions by mixed wheat sour dough starters: Influence of fermentation parameters and dynamics during baking. Food Microbiology. (Estados Unidos). 12:497-507. https://doi.org/10.1016/S0740-0020(95)80134-0

20. GÜL, H.; ÖZÇELIK, S.; SAGDIÇ, O.; CERTEL, M. 2005. Sourdough bread production with lactobacilli and S. cerevisiae isolated from sourdoughs. Process Biochemistry. (Reino Unido). 40(2):691-697. https://doi.org/10.1016/j.procbio.2004.01.044 
21. HAMMES, W.P.; GÄNZLE, M.G. 1998. Sourdough breads and related products. En: Microbiology of Fermented Foods. Springer US. (Estados Unidos). p.199-216. https://doi.org/10.1007/978-1-4613-0309-1_8

22. HASSAN, Y.I.; ZHOU, T.; BULLERMAN, L.B. 2016. Sourdough lactic acid bacteria as antifungal and mycotoxincontrolling agents. Food Science and Technology International. (Estados Unidos). 22(1):79-90. https://doi.org/10.1177/1082013214565722

23. INDECOPI. 2008. Memorias. Disponible desde Internete en: https://repositorio.indecopi.gob.pe/ handle/11724/5801 ?show =full (con acceso 04/02/2020).

24. KARAMAN, K.; SAGDIC, O.; DURAK, M.Z. 2018. Use of phytase active yeasts and lactic acid bacteria isolated from sourdough in the production of whole wheat bread. LWT - Food Science and Technology. 91:557-567. https://doi.org/10.1016/j.lwt.2018.01.055

25. KATINA, K. 2005. Sourdough: A tool for the improved flavour, texture and shelf-life of wheat bread. En: VT'T Publications. Technical Research Center of Finland. (Finlandia). p.1-96.

26. LHOMME, E.; ORAIN, S.; COURCOUX, P.; ONNO, B.; DOUSSET, X. 2015. The predominance of Lactobacillus sanfranciscensis in French organic sourdoughs and its impact on related bread characteristics. Internal J. Food Microbiology. (Paises Bajos). 213:40-48. https://doi.org/10.1016/j.ijfoodmicro.2015.05.010

27. MONTEMURro, M.; CELANO, G.; DE ANGELIS, M.; GOBBETTTI, M.; RIZZELLO, C.G.; PONTONIO, E. 2020. Selection of non-Lactobacillus strains to be used as starters for sourdough fermentation. Food Microbiology. (Estados Unidos). 90:103491.

https://doi.org/10.1016/j.fm.2020.103491

28. MONTVILLE, T.J.; MAT'THEWS, K.R. 2009. Las bacterias del ácido láctico y las fermentaciones de alimentos. En: Microbiología de los alimentos: Introducción (1st ed.). Acribia. (España). 330p.

29. OMEDI, J.O.; HUANG, W. 2016. Soy Sourdough Fermented by Lactic Acid Bacteria Starter (L. Plantarum, and L. Sanfranciscensis) Concentration Effect on Dough Fermentation, Textural and Shelf Life Properties of Wheat Bread. MOJ Food Processing \& Technology. (Hungría). 3(3):327-334. https://doi.org/10.15406/mojfpt.2016.03.00075
30. QUELLEN-FIELD, S. 2012. Biology: Sourdough. En: Culinary Reactions: The everyday chemistry of cooking (1st ed.). Chicago Review Press. (Estados Unidos). p.6.

31. RIZZELLO, C.G.; LORUSSO, A.; MONTEMURRO, M.; GOBBETTI, M. 2016. Use of sourdough made with quinoa (Chenopodium quinoa) flour and autochthonous selected lactic acid bacteria for enhancing the nutritional, textural and sensory features of white bread. Food Microbiology. (Estados Unidos). 56:1-13.

https://doi.org/10.1016/j.fm.2015.11.018

32. RIZZELLO, C.G.; PORTINCASA, P.; MONTEMURRO, M.; DI PALO, D.M.; LORUSSO, M.P.; DE ANGELIS, M.; BONFRATE, L.; GENOT, B.; GOBBETTI, M. 2019. Sourdough fermented breads are more digestible than those started with baker's yeast alone: An in vivo challenge dissecting distinct gastrointestinal responses. Nutrients. (Suiza). 11(12):1-21. https://doi.org/10.3390/nu11122954

33. SÁNCHEZ, M.T. 2003. Planta de elaboración de baguettes semihorneadas congeladas. En: Procesos de elaboración de alimentos y bebidas (1st ed.). Mundi-Prensa. (España). p.105-147.

34. SI, L.W. 2020. Trending foods and beverages. En: Food and Society. Academic Press. (Estados Unidos). p.305-321. https://doi.org/10.1016/b978-0-12-811808-5.00016-7

35. STONE, H.; SIDEL, J.L. 2004. Descriptive Analysis. En: Sensory Evaluation Practices: 3rd ed. Academic Press. (Estados Unidos). p.201-244. https://doi.org/10.1016/B978-0-12-672690-9.X5000-8

36. SWELIM, M.; BASSOUNY, F.; EL-SAYED, S.; HASSAN, N.; IBRAHIM, M. 2014. Sensory Evaluation and Staling of Bread Produced by Mixed Starter of Saccharomyces cerevisiae and L. plantarum. J. Food and Dairy Sciences. (Egipto). 5(4):221-233.

https://doi.org/10.21608/jfds.2014.52765

37. VENTURI, F.; SANMARTIN, C.; TAGLIERI, I.; NARI, A.; ANDRICH, G.; ZINNAI, A. 2016. Effect of the baking process on artisanal sourdough bread-making: A technological and sensory evaluation. Agrochimica. (Italia). 60(3):222-234. https://doi.org/10.12871/00021857201635

38. VENTURI, M.; GUERRINI, S.; VINCENZINI, M. 2012. Stable and non-competitive association of Saccharomyces cerevisiae, Candida milleri and Lactobacillus sanfranciscensis during manufacture of two traditional sourdough baked goods. Food Microbiology. (Estados Unidos). 31(1):107-115.

https://doi.org/10.1016/j.fm.2012.02.011 
39. ZHANG, G.; TU, J.; SADIQ, F.A.; ZHANG, W.; WANG, W. 2019. Prevalence, genetic diversity, and technological functions of the Lactobacillus sanfranciscensis in sourdough: A review. Comprehensive Reviews in Food Science and Food Safety. (Estados Unidos). 18(4):12091226.

https://doi.org/10.1111/1541-4337.12459 